http://dx.doi.org/10.18778/2196-8403.2019.06

\title{
Jenseits von Völkerkerker und friedlichem Mit- einander: Semantisierungen (post-)imperialer Räume und das (süd-)östliche Europa bei MILOš CRNJANSKI und JOSEPH ROTH
}

Dieser Beitrag zeigt anhand ausgewählter Textbeispiele von JOSEPH ROTH und MiLOŠ CRNJANSKI, dass die dargestellten (post-)imperialen Räume in Roths Reportagen über den Westbalkan und CRNJANSKIS lyrischem Roman Tagebuch über Čarnojević weder als Völkerkerker noch - wie es in der kulturwissenschaftlichen Imperien-Forschung der Fall ist - als übernationale Räume jenseits des Nationalen zu begreifen sind. Mit Hilfe von Kosellecks Zeitschichten-Modell und des kulturwissenschaftlichen Konzepts des Palimpsests wird vielmehr gezeigt, dass Nationales und Imperiales auf komplexe Weise aufeinander bezogen sind. Es ist diese Überlagerung sowie die Gleichzeitigkeit des Ungleichzeitigen von Nationalem und Imperialem, aber auch deren Kollision, die den Palimpsest-Charakter des südöstlichen und östlichen Europas in den hier thematisierten Texten ausmacht.

Beyond the "Völkerkerker" and Peaceful Co-existence: Semantization of (Post)Imperial Spaces, and (South)Eastern Europe in MILOŠ CRNJANSKI and JOSEPH RoTH This article, based on selected text extracts from JOSEPH ROTH and MILOŠ CRNJANSKI, demonstrates that the (post)imperial spaces depicted in both ROTH's reports about the western Balkans, and CRNJANSKI's lyrical novel Tagebuch über Čarnojević, can be read as neither Völkerkerker ("prisons of nations"), nor as supranational spaces beyond the national, as is frequently the case in imperial studies within Cultural Studies. Through the lens of Koselleck's model of sediments of time, and the Cultural Studies concept of the palimpsest, the intricate relationships between the national and the imperial are illustrated. This overlap of the national and the imperial and their simultaneous clash constitute the characteristics of a palimpsest of southeastern and eastern Europe in the discussed texts. 


\section{Poza więzieniem narodów i bez pokojowego wspólistnienia: semantyzacja (post)- imperialnych miejsc i (południowo)wschodnia Europa w twórczości MıLošA Crnjanskiego i Józefa Rotha}

Na podstawie wybranych tekstów JÓzEFA RoTHA i MiLOŠA CRNJANSKIEGO artykuł prezentuje tezę, że przedstawione w reportażach ROTHA o zachodnich Bałkanach i w powieści CRNJANSKIEGO Tagebuch über Čarnojević (post)imperialne miejsca nie mają charakteru „więzienia narodów”. Nie można ich także przyporządkować do miejsc ponadnarodowych czy nie mających nic wspólnego z narodem - jak to często jest interpretowane $\mathrm{w}$ badaniach kulturoznawczych poświęconych zagadnieniom związanym z rozwojem imperiów. Za pomocą modelu wielowarstwowości czasu historycznego Reinharta Kosellecka oraz kulturoznawczej koncepcji palimpsestu ukazane zostaje, że pojęcia narodowości i imperialności są ze sobą powiązane. Nakładanie się na siebie tych zjawisk oraz nierównoczesna jednoczesność narodowości i imperialności, także ich opozycja wobec siebie tworzy palimpsestyczny charakter południowo-wschodniej i wschodniej Europy przedstawiony w analizowanych tekstach.

Der vorliegende Beitrag setzt sich mit Semantisierungen (vgl. OROSz 2018:7-35) des (süd-) östlichen Europas am Beispiel ausgewählter Texte von MILOŠ CRNJANSKI und JOSEPH ROTH auseinander. Unter Semantisierung wird hier die Art und Weise verstanden, in der das (süd-)östliche Europa als (post-)imperialer Raum konstruiert und bewertet wird. Mit imperial ist jener weltgeschichtliche Abschnitt des 19. und 20. Jahrhunderts gemeint, in dem die drei großen Landimperien, das Habsburger-Reich, das Osmanische Reich und das Russische Zarenreich noch nicht von der Landkarte verschwunden waren. (Post-)Imperial hingegen bezeichnet die Zeit nach dem Ersten Weltkrieg. Die hier genannten Kontinentalimperien gingen zwar im Zuge des Ersten Weltkriegs endgültig unter, ihr Erbe (vgl. TODOROVA 2002:470-492) hingegen blieb, wie auch immer es im Einzelnen zu bewerten sein mag, weiterhin bestehen.

Die Frage, wie das Erbe der untergegangen Kontinentalimperien im (süd-)östlichen Europa in literarischen Texten jeweils dargestellt wird, kann nicht pauschal, d. h. mit Hilfe von Generalthesen beantwortet werden. Dafür sind die Formen der Semantisierung sowohl imperialer als auch postimperialer Räume zu vielschichtig und heterogen. Dies gilt es im Folgenden am Beispiel ausgewählter Texte JOSEPH ROTHS und MiLOŠ CRNJANSKIS zu zeigen.

Das Interesse an Imperien, imperialen und (post-)imperialen Räumen hat derzeit Hochkonjunktur (vgl. GROB / PREVIŠIĆ 2014; BOBINAC / CHOVANEC 2018). Vor dem Hintergrund neuerer geschichtswissenschaftlicher Ansätze, die zunehmend globale Verflechtungen und damit auch transnationale Fragen und Problemkonstellationen in den Mittelpunkt ihrer Forschungen rücken (vgl. PATEL 2008:67-89), 
sind Imperien inzwischen zu einem bevorzugten Forschungsgegenstand avanciert (vgl. OSTERHAMMEL 2016; LEONHARD / HIRSCHHAUSEN 2012; BURBANK / COOPER 2010; OSTERHAMMEL 2006; MÜNKLER 2005). Der Konstanzer Globalhistoriker Jürgen Osterhammel weist in diesem Kontext auf den von der Nationalgeschichtsschreibung nicht zufällig vernachlässigten Sachverhalt hin, „dass die gesamte Historiographie der Welt vor dem Aufkommen des Nationalstaates im Regelfall mit Imperien zu tun“(OSTERHAMMEL 2006:4) hat.

Doch nicht nur die Geschichtswissenschaften, sondern auch eine kulturwissenschaftlich argumentierende Literaturwissenschaft (vgl. GROB / PREVIŠIĆ 2014) widmet sich in Auseinandersetzung mit literarischen Texten der Frage, wie (post-) imperiale Räume in der Literatur dargestellt werden. Anders als geschichtswissenschaftliche Arbeiten (vgl. OSTERHAMMEL 2006) sind kulturwissenschaftliche Studien stark darum bemüht, (post-)imperialen Räumen positive Seiten abzugewinnen. Im Gegensatz zu homogenen Nationalstaaten, so die Kernthese, böten Imperien vielfache Chancen und Möglichkeiten für kulturelle Transfers (vgl. BALEVA / PREVIŠIĆ 2018). Besonders akzentuiert wird dabei, dass das Miteinander unterschiedlicher Ethnien und Religionen nicht per se zu Konflikten und Kriegen geführt habe (vgl. GROB / PREVIŠIĆ 2014:7). Diesem Befund kann hier schlechterdings nicht widersprochen werden.

In der ethnischen und religiösen Heterogenität (post-)imperialer Räume die Ursache von Krieg und Gewalt suchen zu wollen, ist in der Tat mehr als fragwürdig. Erstens unterstellt eine derartige Sichtweise historischen Prozessen Zwangsläufigkeit, zweitens impliziert sie, dass ein weitestgehend friedliches Zusammenleben unterschiedlicher Ethnien und Religionen an und für sich unmöglich sei. Diesem Vorurteil wird zurecht mit folgendem Gegenargument begegnet:

Und doch waren es entgegen der Wahrnehmung der Zeit nicht primär die explosiven Kräfte dieser multiethnischen, ja in gewissem Sinne - da Nation noch keineswegs mit Territorialstaat deckungsgleich sein musste - multinationale Gebilde, die Europa besonders in der ersten Hälfte des 20. Jahrhunderts mörderischen Auseinandersetzungen ausliefern sollten. Vielmehr entfaltete das territorialisierte nationale Denken selbst das größte Konfliktpotential. (GROB / PREvišIĆ 2014:7)

Imperiale Gebilde können auch schon deshalb nicht verallgemeinernd als Völkerkerker betrachtet werden, weil es Ihnen nicht allein mithilfe von Gewaltanwendung und Unterdrückung hätte gelingen können, Jahrhunderte zu überdauern. Ihre Fähigkeit, Heterogenität mittels einer ,politics of difference“ (vgl. BURBANK / COOPER 2010:1-22) effizient zu handhaben und zu balancieren, spielte hier eine nicht zu unterschätzende Rolle. 
Einer kulturwissenschaftlich orientierten Literaturwissenschaft erscheinen Imperien jedoch vor allem aufgrund ihrer „ethnisch-religiösen Heterogenität“", aber auch aufgrund ,ihre[r] überregional ausgerichteten administrativen oder kommunikativen Raumstrukturen“ (GROB / PREVIŠIĆ 2014:12) als positive und in intellektueller Hinsicht attraktive Gegenmodelle zu Nationalstaaten. Die Geschichtswissenschaften sind hier weitaus vorsichtiger. Während das Phänomen des Imperialismus in kulturwissenschaftlichen Beiträgen bezeichnenderweise keinerlei Beachtung findet, wird es in der geschichtswissenschaftlichen Imperien-Forschung als integraler Bestandteil imperialer Machtpolitik reflektiert und nach wie vor erforscht (vgl. OSTERHAMMEL 2006:56-67).

$\mathrm{Zu}$ kurz kommt in kulturwissenschaftlichen Perspektiven auf (post-)imperiale Räume die Tatsache, dass nicht nur Nationalstaaten, sondern auch Imperien Exklusion und Unterdrückung ethnisch-religiöser Minderheiten betrieben, indem sie sich während des 19. und 20. Jahrhundert nationaler Herrschaftsstrategien bedienten (vgl. HIRSCHHAUSEN / LEONHARD 2011). Insbesondere in Krisenund Kriegsphasen, in denen Loyalitäten auf dem Spiel stehen und überall Verrat vermutet wird, lässt sich beobachten, wie repressiv auch Imperien gegen ihre ethnisch und religiös heterogene Bevölkerung vorgehen konnten (vgl. BORODZIEJ / GÓRNY 2018:240-263).

Galten Imperien lange Zeit als Völkerkerker, so werden sie in jüngster Zeit, insbesondere in kulturwissenschaftlich argumentierenden Studien, zu übernationalen Möglichkeitsräumen deklariert, die als Modelle für ein zukünftig geeintes Europa dienen könnten (vgl. PREVIŠIĆ 2014:25-42). Dahinter verbirgt sich jedoch, ganz ähnlich wie im Falle des nicht minder einseitigen Narrativs, wonach Imperien Völkerkerker gewesen seien, eine eindeutige politische Stoßrichtung. Das in den Kulturwissenschaften zu beobachtende Bestreben, Imperien aufzuwerten, ist maßgeblich dadurch motiviert, dass die heutige Europäische Union in die Krise geraten ist, so dass nach politischen Ordnungsmodellen jenseits des Nationalstaates gesucht wird. Imperien erscheinen auch vor diesem Hintergrund als positives Gegenmodell zum Nationalstaat.

So fordert der Politik- und Kulturwissenschaftler Herfried Münkler in seinem Buch Imperien. Die Logik der Weltherrschaft - Vom Alten Rom bis zu den Vereinigten Staaten (vgl. MüNKLER 2005) nicht von ungefähr, dass Europa in Zukunft eine Art Imperium werden müsse (vgl. MÜNKLER 2005:253f.). Sein Kernargument, mit dem er diesen Appell zu untermauern sucht, ist dabei, dass imperiale Räume im Gegensatz zu Nationalsaaten von halbdurchlässigen Grenzen durchzogen waren. 
Die für Staaten typische Grenzziehung ist scharf und markant; sie bezeichnet den Übergang von einem Staat zu einem anderen. Solche präzisen Trennungslinien sind im Falle von Imperien die Ausnahme. (MÜNKLER 2005:16)

Daraus zieht er folgende, bereits erwähnte Schlussfolgerung:

Europa wird, wenn es sich nicht überfordern und schließlich scheitern will, dieses imperiale Modell der Grenzziehung übernehmen müssen. Im Prinzip ist ihm eine solche Ordnung eingeschrieben, verlaufen doch die Außengrenzen der EU anders als die des Schengenraumes und die wiederum anders als die der Eurozone. Dieses Modell gilt es weiterzuentwickeln, um die europäischen Außengrenzen stabil und elastisch zugleich zu machen. Das schließt Einflussnahmen auf die Peripherie ein, die eher imperialen als zwischenstaatlichen Vorgaben ähneln. Europas Zukunft wird darum ohne Anleihen beim Ordnungsmodell der Imperien nicht auskommen. (vgl. MÜNKLER 2005:253f.)

Auch wenn Imperien, wie Münkler zurecht betont, nicht allein auf Imperialismus und Ausbeutung ihrer Peripherien reduziert werden können (vgl. MÜNKLER 2005:11-30), wäre es gleichwohl ahistorisch, versuchte man jene Integrationsstrategien, zu denen Imperien griffen, um verschiedene Ethnien und Religionen an sich zu binden, auf die heutige Zeit übertragen zu wollen. Münklers politikwissenschaftlicher Ansatz, aber auch die kulturwissenschaftliche ImperienForschung, die sich maßgeblich auf Münklers Thesen stützt, erscheinen daher nur bedingt geeignet, die vielfältigen Darstellungsweisen und Deutungen imperialer und postimperialer Räume in literarischen Texten in ihrer Widersprüchlichkeit und Widerspenstigkeit zu erfassen. Um präziser beschreiben und analysieren zu können, wie das (süd-)östliche Europa bei CRNJANSKI und ROTH jeweils semantisiert wird, erscheint es sinnvoller und zielführender, danach zu fragen, von wem die dargestellten (post-)imperialen Räume bevölkert werden. Wie sehen die Interaktionen zwischen den jeweiligen Ethnien und Religionen in derart verfassten Räumen konkret aus? Bevor aber diesen Fragen am Beispiel literarischer Texte nachgegangen wird, müssen einige begriffsklärende sowie methodisch-erkenntnistheoretische Bemerkungen vorangeschickt werden. Diese betreffen die Begriffe ,Ostmitteleuropa', ,Südosteuropa', ,Balkan', aber auch die methodisch-theoretischen Konzepte, mit deren Hilfe die Texte ROTHS und CRNJANSKIS analysiert werden.

\section{Begriffsklärende Überlegungen}

Was unter ,Ostmitteleuropa ‘, ,Zentraleuropa', aber auch dem ,Balkan` oder ,Südosteuropa' verstanden wird, ist in den Geschichts- und Kulturwissenschaften 
höchst umstritten. Erschwerend kommt hinzu, dass selbst in den Geschichtswissenschaften keine Einigkeit darüber herrscht, was eigentlich gemeint ist. Die Semantik der Begriffe ist daher alles andere als eindeutig. Die hier vorgenommenen begriffsklärenden Überlegungen, die nicht beanspruchen, eine Begriffsgeschichte zu sein, beziehen sich vor allem auf die Verwendung des Begriffs ,Balkan“. Das ist der Tatsache geschuldet, dass dieser Raum, nicht zuletzt wegen des Jugoslawien-Kriegs der neunziger Jahre im 20. Jahrhundert, die größten pejorativen Vorstellungen und Bilder in den Köpfen westlicher Beobachter*innen erzeugt.

Die Bezeichnungen ,Balkan“ und ,Südosteuropa“ werden einerseits synonym verwendet, andererseits fungiert ,Südosteuropa ‘ als vermeintlich wertneutraler Begriff für den als genuin rückständig und gewalttätig-atavistisch geltenden Balkan. Eine, wenn nicht die entscheidende Frage, die sich bei dem Versuch stellt, den Balkan zu definieren, ist jene, wie er zum vorwiegend ,christlichabendländisch' geprägten Westen steht. Doch lässt sich der Balkan tatsächlich als ein Raum definieren, der sich vom restlichen Europa gänzlich unterscheidet? Entscheidet man sich dafür, den Balkan mit Hilfe eines festen Merkmal-Bündels zu beschreiben, dann sind es meist folgende Aspekte, die den Balkan zu einem im Vergleich zu anderen Regionen Europas vermeintlich außer-ordentlichen Raum machen: Rückständigkeit, verspätete Nationsbildung, Überfluss an Mythen sowie das islamisch-osmanische und orthodox-byzantinische Erbe (vgl. SuNDHAUSSEN 1999:638-651).

$\mathrm{Zu}$ dem gängigen Bild, wonach der Balkan ein Raum sei, den ein im Vergleich zu anderen Teilen Europas ungewöhnlich hohes Maß an Gewalt und Brutalität kennzeichne, hat zweifelsohne insbesondere der Jugoslawien-Krieg der neunziger Jahre beigetragen. Nicht von ungefähr wurde er fälschlicher-, aber dadurch auch bezeichnenderweise immer wieder als Balkankrieg bezeichnet (vgl. BALEVA / PREVIŠIĆ 2018:7-24). Folgt man dieser vor allem den öffentlichen (vgl. SuNDHAUSSEN 2007 und BALEVA / PREVIŠIĆ 2018:18) Diskurs bestimmenden Sicht, die sich zum Teil auch in der Geschichtswissenschaft findet (vgl. SUNDHAUSSEN 1999:638-651), wonach sich der Balkan in seiner historischen Entwicklung vom Westen substanziell unterscheide, so ergeben sich zwangsläufig Probleme erkenntnistheoretischer Natur (vgl. TODOROVA 2002:488). Sie resultieren vor allem daraus, dass Räume und Kulturen als in sich geschlossen und homogen betrachtet werden.

Bei solchen Versuchen, Räume zu definieren, geht es somit auch stets um die Frage, welcher Kulturbegriff, aber auch darum, welcher erkenntnistheoretische Blickwinkel den jeweiligen Raumkonstruktionen zugrunde liegt. Folgt man 
der Annahme, dass Rückständigkeit und Gewalt spezifische Merkmale des Balkans sind, stellt sich bei genauerem Hinsehen heraus, dass hier immer noch das Paradigma der Nationalgeschichtsschreibung leitend ist. Merkmale, die aus nationaler Perspektive, wenn auch vermeintlich, als spezifisch balkanisch erscheinen mögen, verlieren viel von ihrer Besonderheit, ordnet man sie in einen größeren historischen, d. h. gesamteuropäischen oder globalgeschichtlichen Kontext ein (vgl. TODOROVA 2003:227-253).

Für einen derartigen methodisch-erkenntnistheoretischen Zugriff plädiert Maria Todorova (vgl. TODOROVA 2007:89-111). Sie zeigt, wie wissenschaftlich und erkenntnistheoretisch fragwürdig es ist, den Balkan, aber auch das östliche Europa als Räume zu betrachten, die von gesamteuropäischen und globalen Entwicklungen größtenteils, wenn nicht gar völlig abgeschnitten waren. Und es stellt sich hier nicht zuletzt die Frage, ob das, was wir oft unreflektiert als den Westen bezeichnen, tatsächlich so homogen ist (vgl. TODOROVA 2007:21).

Anstatt den Balkan am Westen als dessen Norm und Vorbild zu messen, haben neuere Forschungen in überzeugender Weise gezeigt, dass dieser Raum ,geografisch, kulturell und historisch wichtige Bruchstellen“ (BALEVA / PREVIŠIĆ 2018:13) mit dem gesamteuropäischen, aber auch dem globalen Kontext aufweist. Ähnlich umstritten, wenn auch aus anderen Gründen, sind Begriffe wie ,Mitteleuropa' und ,Ostmitteleuropa'. Während Ostmitteleuropa stark strukturgeschichtlich konnotiert ist, diente der Mitteleuropa-Begriff der Legitimation deutscher Kriegsziele im Osten (vgl. THER 2006). Strukturgeschichtliche RaumDefinitionen wie Ostmitteleuropa haben den Nachteil, dass sie die Grenzen zwischen dem östlichen und dem südöstlichen Europa zu scharf ziehen, so dass kulturelle Wechselbeziehungen kaum in den Blick rücken (vgl. THER 2006).

Und auch ein vermeintlich neutraler Begriff wie ,Südosteuropa' ist bei genauerer Betrachtung negativ besetzt. Schließlich wurde dieser Begriff von den Nationalsozialisten in ihren Europaplänen verwandt (vgl. BALEVA / PREVIŠIĆ 2018:13). Für eine Beschreibung der vielfältigen Spielarten des Zusammenlebens und der Kulturkontakte auf den Gebieten Ostmittel-, Zentral und Südosteuropas, wie sie u.a. in Texten von JOSEPH ROTH und MILOŠ CRNJANSKI dargestellt und durchgespielt werden, bietet es sich zunächst an, von Maria Todorovas Kategorie des östlichen Europas auszugehen. Todorova versteht darunter „einen locker gefügten, konventionellen historisch-geographischen Raum, der neben Ostmitteleuropa auch Südosteuropa bzw. den Balkan umfasst“" (TODOROVA 2007:21). Die historischen Prozesse, die im östlichen und westlichen Europa während des 19. Jahrhunderts stattgefunden haben, lassen sich, so Todorova, am präzisesten 
beschreiben, geht man von einer ,relative[n] Synchronität innerhalb eines longue durée-Rahmens“ aus (TODOROVA 2007:19). Sie widerspricht damit der weit verbreiteten These, wonach jegliche Entwicklungen, die im östlichen Europa stattfanden, als Nachahmung westeuropäischer Vorbilder zu betrachten seien (vgl. TODOROVA 2007).

Was Todorova unter der Bezeichnung ,östliches Europa' versteht, entspricht konzeptionell am ehesten Moritz Csákys Zentraleuropa-Begriff. Obgleich Csákys mit Zentraleuropa keinen streng abgrenzbaren geographischen Raum meint, zählt er sehr wohl all jene Länder zu ihm, die einst Teil ÖsterreichUngarns waren (vgl. CSÁKY 2010:37-68). Nichtsdestotrotz will Csáky unter Zentraleuropa keinen geografisch eindeutig eingrenzbaren Raum verstanden wissen. Es geht ihm vielmehr um ein ,intellektuelle[s] Konzept“", das es gestattet, „Kulturen als dynamische und offene Kommunikationsräume“ (CSÁKY 2009:61) zu denken. Dies gilt ebenso für Todorovas Definition des östlichen Europas. Csákys Zentraleuropa-Begriff suggeriert dennoch eine geographische Mittelstellung, die so nicht intendiert ist, weil sie dem Kultur- und Raum-Verständnis Csákys widerspricht.

In Anlehnung an Maria Todorova wird hier der Bezeichnung, (süd-)östliches Europa' daher der Vorzug gegeben. Nur vom östlichen Europa zu sprechen, würde zwangsläufig zu Missverständnissen führen, weil ROTHS Reportagen über den Westbalkan ebenso unter den Terminus ,östliches Europa' fallen würden wie CRNJANSKIS Darstellung Galiziens. Der Terminus ,(süd-)östliches Europa ‘ erscheint hier am besten geeignet, weil mit ihm sowohl das östliche als auch das südöstliche Europa begrifflich auf einen Nenner gebracht werden können.

\section{Methodisch erkenntnistheoretischer Rahmen: Zeitschichten- und Palimpsest-Metapher zur Beschreibung von Raum-Zeit- Konstellationen}

Für eine Analyse und Beschreibung des Verhältnisses zwischen / von Imperialem und Nationalem (vgl. LEONHARD / HIRSCHHAUESEN 2011) anhand von CRNJANSKIS Tagebuch über Čarnojević und ROTHS Reportagen über den Westbalkan bietet sich eine Orientierung an den kultur- und geschichtswissenschaftlichen Metaphern des Palimpsests (vgl. OsthuEs 2017:37-53) und der Zeitschichten (vgl. KOSELLECK 2015) an.

Mit dieser Verschränkung geschichts- und kulturwissenschaftlicher Ansätze wird eine methodische Präzisierung rein kulturwissenschaftlicher Ansätze angestrebt. 
Damit soll ein möglichst offener Blick auf die Verfasstheit (post-)imperialer Räume ermöglicht werden, der sie weder als Völkerkerker zu verdammen sucht noch von vornherein als Orte jenseits nationaler Antagonismen und Verwerfungen begreift. Der Geschichtstheoretiker Reinhart Koselleck hat die im Rahmen seiner begriffsgeschichtlichen Studien geprägte Zeitschichten-Metapher wie folgt definiert:

$\mathrm{Da} ß$ auch Geschichte eine räumliche Konnotation zuläßt, nämlich die, Schichten $\mathrm{zu}$ enthalten, mag als Wortspielerei abgetan werden. Aber die verräumlichende Metapher, die den Zeitbegriff pluralisiert, hat einen Vorteil für sich. Zeitschichten verweisen, wie ihr geologisches Vorbild, auf mehrere Zeitebenen verschiedener Dauer und unterschiedlicher Herkunft, die dennoch gleichzeitig vorhanden und wirksam sind. Auch die Gleichzeitigkeit des Ungleichzeitigen, eines der aufschlußreichsten historischen Phänomene, wird mit Zeitschichten auf einen gemeinsamen Begriff gebracht. (KOSELLECK 2015:9f.)

Die Zeitschichten-Metapher dient Koselleck zur Beschreibung der Mehrschichtigkeit historisch-kultureller Prozesse. Sie hat den Vorteil, dass sie geschichtliche Prozesse nicht als teleologisch-linear denkt. Im Gegensatz zu einem derartigen Verständnis von Zeit impliziert das Zeitschichten-Modell, dass sich historische Prozesse auf ,mehrere[n] Zeitebenen verschiedener Dauer und unterschiedlicher Herkunft“ abspielen, ,die dennoch gleichzeitig vorhanden und wirksam sind“ (KOSELLECK 2015:9). Geschichtliche Verläufe sind an und für sich dadurch gekennzeichnet, dass sich neuere Entwicklungen ankündigen und bemerkbar machen können, ohne indes vorher stattgefundene Prozesse sofort abzulösen. Das meint Koselleck mit der Gedankenfigur der Gleichzeitigkeit des Ungleichzeitigen.

Es ist diese anti-teleologische Geschichtsauffassung, die die Zeitschichten und die Palimpsest-Metapher teilen. Beide Metaphern ermöglichen es, jene divergierenden historischen und kulturellen Stränge, die sich in imperial und postimperial geprägten Räumen abgelagert haben, zu beschreiben und zu analysieren. Für die Interpretation literarischer Texte bedeutet dies, dass danach gefragt werden muss, wie diese unterschiedlichen historisch-kulturellen Schichten in den Texten jeweils semantisiert, d. h. gewichtet und beurteilt werden. Das (süd-)östliche Europa vor und auch noch nach dem Ersten Weltkrieg ist insofern als eine Art Palimpsest zu denken, als es sich um einen Raum handelt, in dem sich im Laufe der Jahrhunderte byzantinische, jüdische, islamische, aber auch katholische Spuren wie Gesteinsschichten übereinandergelegt haben.

Wesentlich ist hier jedoch, welche Stränge in den zu untersuchenden Zeitabschnitten dominierten. Denkt man (süd-)östliche Europa als Palimpsest, so hat dies den entscheidenden Vorteil, dass dadurch ,vermeintlich Gegensätzliches und Ungleichzeitiges" (OSTHUES 2017:43) zueinander in Beziehung gesetzt werden kann. Nation und Imperium, Imperiales und Nationales können - zumindest 
auf den ersten Blick - als derartige Gegensätze gelten. Während Nationen ihren ,Ursprung“ im 19. Jahrhundert haben, reicht die Entstehung imperialer Gebilde bis in die Antike und das Mittelalter zurück. Hinsichtlich der Gedankenfigur der Gleichzeitigkeit des Ungleichzeitigen historischer Prozesse ist vor dieser Folie bedeutsam, dass im 19. und 20. Jahrhundert ein Neben-, aber auch Gegeneinander imperialer und nationaler Strukturen und Entwicklungen zu beobachten ist. Im Unterschied zum sogenannten spatial turn (vgl. SCHLÖGEL 2003:308 und OROSZ 2018:16-35), der den Primat des Raumes vor der Zeit ausrief und postulierte, vermag die Zeitschichten-Metapher der Tatsache Rechnung zu tragen, dass bei der Beschreibung historischer Prozesse Zeit und Raum stets in gleichberechtigter Weise aufeinander bezogen sind. Koselleck illustriert dies, wie aus dem Eingangszitat hervorgeht, am Beispiel des Begriffs Geschichte selbst. Geschichte impliziere einerseits, dass etwas geschieht, indem es sich in der Zeit ereignet, aber auch die Vorstellung der Schichtung und Überlagerung älterer und neuerer geschichtlicher Verläufe. Überträgt und bezieht man die Palimpsestund Zeitschichtenmetapher nun auf das (süd-)östliche Europa des 19. und 20. Jahrhunderts, erweist sich, dass die Gleichzeitigkeit des Ungleichzeitigen unter Umständen darin zu sehen ist, dass Nationales und Imperiales keine absoluten Gegensätze darstellen (vgl. HIRSCHHAUSEN / LEONHARD 2011:9-34). Das wird im Folgenden am Beispiel von ROTHS Reportagen über Südslawen und MILOŠ CRNJANSKIS 1921 erschienenem Roman Tagebuch über Čarnojević gezeigt.

Auf den ersten Blick mag es methodisch problematisch, wenn nicht gar unzulässig erscheinen, Reportagen mit einem fiktionalen Text vergleichen zu wollen. Vergegenwärtigt man sich aber, dass ROTHS Reportagen die Grenze zwischen Fiktion und Wirklichkeit immer wieder einebnen, wird dieser an und für sich berechtigte Einwand hinfällig. Den spezifischen Charakter der ROTH'SCHEN Reportagen beschreibt Achim Küpper wie folgt:

Ist die Reportage an sich schon eine äußerst subjektiv und künstlerisch geprägte Stilform journalistischer Berichterstattung, so lösen sich bei Roth [...] die Grenzen zwischen Reportage und Erzählung, zwischen dokumentarischer und literarischer Aussageweise gänzlich auf. (KÜPPER 2010:100f.)

Aus diesem Befund zieht Küpper die „texttheoretische Konsequenz“ (KÜPPER 2010:101), dass ,die Stimme des Berichtenden“ in ROTHS Reportagen ,grundsätzlich von der des Autors“ (vgl. KÜPPER 2010:101) unterschieden werden müsse. Reportage und Romans sind im Werk ROTHS deshalb verwandte Textgattungen, weil auch ROTHS Reportagen über eine Erzählstimme verfügen, die, wie Küpper zeigt, nicht identisch ist mit dem Autor ROTH (vgl. KÜPPER 2010). Es bietet sich daher geradezu an, ROTHS Reportagen und CRNJANSKIS Roman auf die 
hier aufgeworfene Frage nach der Semantisierung des (süd-)östlichen Europas hin zu untersuchen. Zudem hat die ROTH-Forschung hinlänglich darauf hingewiesen, dass durchaus Parallelen zwischen den journalistischen Arbeiten und dem fiktionalen Werk RoTHS bestehen (vgl. WALTL 2016:17-30; SÜLTEMEYERVON LIPS 2016:31-59). Die journalistischen Städtebilder ROTHS sind somit Teil jener „,imaginären Topographie“ (FUNK 2012:59), die er in seinen Romanen entfaltet.

\section{Fallbeispiele: MILOŠ CRNJANSKI und JOSEPH ROTH}

Wie wird das Verhältnis von ethnischen und religiösen Minderheiten zum (post-)imperialen Raum bei ROTH und CRNJANSKI dargestellt? Während sich CRNJANSKIS Tagebuch über Čarnojevic noch größtenteils auf die imperiale Phase, d. h. die Zeit während des Ersten Weltkriegs bezieht, wird in ROTHS Reportagen über den Westbalkan die Zeit nach dem Ersten Weltkrieg und damit die (post-)imperiale Phase adressiert. Der 1893 in der ungarischen Kleinstadt Csongrád als Sohn eines serbischen Beamten geborene Schriftsteller MILOŠ CRNJANSKI trauerte im Gegensatz zu JOSEPH ROTH der Habsburger Monarchie alles andere als nach. CRNJANSKI wurde 1914 rekrutiert und musste als Soldat der Habsburgermonarchie an der galizischen Front gegen das Russische Zarenreich kämpfen. Er kam als einer der wenigen Überlebenden seines Bataillons davon. Für die Art und Weise, in der das östliche Europa in seinem Roman Tagebuch über Čarnojevic semantisiert wird, ist die Kriegserfahrung CRNJANSKIS durchaus relevant. Es versteht sich von selbst, dass es methodisch naiv wäre, Figur und Autor gleichzusetzen.

Dennoch ist es schwerlich ein Zufall, dass sich der Protagonist des Romans gleich zu Beginn als herumirrender, ja vagabundierender Soldat zu erkennen gibt, der von der galizischen Front zurückkehrte, von jenen, wie es heißt, „, blutigen, rote[n], warmen Wäldern“, den ,unüberschaubaren polnischen Wäldern“ (CRNJANSKI 1993:9). Über sein Soldaten-Dasein heißt es: „Ich bin Soldat, oh, keiner weiß, was das bedeutet" (CRNJANSKI 1993:9).

Dieses Unaussprechliche, von dem hier die Rede ist, wird den Leserinnen und Lesern des Romans immer wieder in eindringlich-drastischen Bildern vor Augen geführt. Bereits auf den ersten Seiten finden jene Soldaten Erwähnung, die aus den Wäldern Galiziens nie wieder zurückkehren sollten. Dem Schrecken des Krieges wird eine subjektiv-lyrische Naturbetrachtung entgegengesetzt. Der 
Protagonist liebt das Leben nur deshalb, weil er es zu schätzen weiß, dass er überlebt hat:

Ich bin verliebt in die Gewässer hier, die Bäume hinter den Festungsmauern, die sich zwischen den gelben und grünen Pfützen verlieren, wo das Gras so weich, versengt und warm ist. Und ich liebe mein Leben mit der Faszination, die ich voriges Jahr empfand, als ich aus diesen schmutzigen, jungen polnischen Wäldern zurückkehrte, wo so viele geblieben sind, zerfetzt und blutig mit zerschmettertem Schädel. (CRNJANSKI 1993:9)

Das östliche Europa zur Zeit des Ersten Weltkriegs wird bei CRNJANSKI u.a. als eine Art Schlachthaus semantisiert, in dem der Tod allgegenwärtig ist:

Im Wald wurde so grausam geschlachtet. Leute kamen angerannt, außer Atem, schreckerfüllt - sie wollten fliehen. Wieder gruben wir uns am Waldrand ein. Ich liege und atme, atme schnell; aus der Nase fließt mir langsam das Blut. Piijjuu fitsch...schlägt ein Geschoß neben meinem Kopf in die Erde. (CRNJANSKI 1993:22f.) Zurück bleiben in den ,russischen Gräben“ Soldaten „,mit eingeschlagenen Schädeln, aber auch „,[v]erlauste, ungewaschene, kraftlose, gelbe, stinkende Männer, einige noch lebend mit stupidem Blick, im Begriff, den Geist aufzugeben. Einer von uns erblickte seinen Bruder, der da zwischen ihnen lag. Er erzitterte und schrie gräßlich“(CRNJANSKI 1993:23).

Und trotz allem sind die Soldaten gezwungen weiterzumarschieren: „Das Bataillon stampfte und stapfte weiter durch die Wälder...“(CRNJANSKI 1993:23). Was durch das Verb „stampfen“ angesprochen und im Text in vielfältig codierter Weise durchgespielt und konkretisiert wird, ist das Moment der Mobilität und des Reisens, wobei der Begriff des Reisens hier im weitesten Sinne zu verstehen ist. Die verschiedenen Spielarten des Reisens im Tagebuch über Čarnojević sind daher am ehesten unter den Obergriff der Fortbewegung im Raum zu subsumieren. Bezüglich der Frage, wie imperiale Räume semantisiert werden, ist das Phänomen der Mobilität besonders aufschlussreich. Gilt es doch als eines der wesentlichen, wenn nicht als das wesentliche Merkmal imperialer, aber auch postimperialer Räume. Kulturwissenschaftliche Studien beurteilen das Phänomen der Mobilität in (post-)imperialen Räumen als durchweg positiv:

Mobilität ist in ihren vielfältigen Formen sicher eines der aufschlussreichsten Paradigmen, imperiale Raum- und Grenzbildungen zu verfolgen. Nichts macht Grenzen so sichtbar, wie sie zu überschreiten, sei dies nun physisch oder mental; so sind Mobilitätsformen in besonderer Weise geeignet, die Vielschichtigkeit imperialer Grenzen sichtbar zu machen. An den Formen von Mobilität erweisen sich die Beziehungen zwischen Peripherie und Zentrum oder diejenigen zwischen verschiedenen Peripherien, sei es als geographische und soziale Mobilität im Zuge von Modernisierung und Urbanisierung, als Potential für imaginative Raumentwürfe, als individuelle Karrierebildung oder schlicht als Alltagserfahrung. Gerade 
auch biographisch-soziale Mobilität ist in imperialen Landschaften meist mit räumlicher Bewegung verbunden (vgl. GROB / PREvIŠIć 2014:12f.).

Die Mobilitätserfahrungen, die der Protagonist Tagebuch über Čarnojević macht, sind indes mitnichten durchgängig positiv konnotiert. Dies wirkt sich wiederum maßgeblich auf die Konstruktion und Bewertung des (süd-)östlichen Europas aus. Die Reise- und Mobilitätserfahrungen des Protagonisten vor und während des Krieges lassen sich cum grano salis als freiwillige versus erzwungene Fortbewegung im Raum charakterisieren. Der Protagonist, so erfahren wir, ist schon in seiner Kindheit viel herumgekommen, weil die Mutter leidenschaftlich reiste. „Meine Mutter reiste oft und gern“ (CRNJANSKI 1993:16). Es handelt sich um eine Lebensweise, die sich geradezu permanent auf Reisen und dadurch auch stets in der ,Fremde', fernab der Heimat abspielt: „Man lebte immer in der Fremde. Man zog um, zog um. Und lungenleidend starb man zu Hause in der Heimat" (CRNJANSKI 1993:37).

In dem Verweis darauf, dass ein solches Leben letztlich mit einem schmerzhaften Tod in der ,Heimat' endet, liegt die ganze Tragik dieser zweifelsohne höchst dynamischen Existenzweise begründet. Während wir es bei dieser Art des Reisens jedoch, obgleich auch sie nicht gänzlich positiv ausfällt, mit einer freiwilligen Form der Mobilität zu tun haben, ist die Fortbewegung während des Krieges eine erzwungene, der sich der Protagonist nolens volens zu fügen hat.

Nicht von ungefähr ist von einer schweren „Hetzjagd durch Syrmien und die versengte Sava Ebene“ (CRNJANSKI 1993:27) die Rede. Diese Verfolgungsjagd durch einen Teil des südöstlichen Europas erscheint jedoch im Vergleich zu den Bewegungen der Soldaten an der galizischen Front als weitaus weniger schrecklich und ermüdend. In Galizien müssen die Soldaten „ein Meer von Morast“ (CRNJANSKI 1993:27) durchschreiten. Diese ,unendliche[n], schlammige[n] Wege" (CRNJANSKI 1993:29) führen letztlich dazu, dass die Konturen der Landschaft verschwimmen.

Das Ergebnis ist, dass den Soldaten räumliche Orientierung unmöglich gemacht wird, so dass sie nicht einmal mehr wissen, wo sich der Feind befindet. „In den Wolken der gelben Erde und in dem Morast, der hochspritzte, verschwand die russische Stellung“ (CRNJANSKI 1993:27). Der Eindruck der durch den Krieg verursachten Orientierungslosigkeit und des allgemeinen Durcheinanders wird zusätzlich durch das Bild einander jagender und wild umherirrender Massen potenziert und regelrecht auf die Spitze getrieben:

Überall jagen Horden einander, streifen umher. Da, wie das Vieh laufen sie auf Horodenka zu, wie das Vieh verrecken sie. Schau sie an, wie sie sich untereinander abschlachten, im Höllenlärm der Wälder und des Morastes, in der Agonie 
blutiger Hände und Gedanken. Millionen halten sich in den Städten bereit wo Meere babylonischer Türme stehen, wo der Sturm aus Stahl ist und das Donnern aus Eisen, bei den riesigen Häfen voll mit Schiffen. Und die monströse Freiheitsstatue steht da und schaut über den Ozean, wo unter dem makedonischen Himmel die Fenster blitzen, wo überall die Armut hervorschaut, dort erklingt ein Lied. (CRNJANSKI 1993:38)

Dass der (post-)imperiale Raum bei CRNJANSKI größtenteils negativ konnotiert ist, lässt sich auch daran ablesen, dass das im Tagebuch dargestellte östliche Europa von „Scharen einsamer Frauen, Horden betrügerischer Händler, Horden von Arbeitern, Scharen von Kranken und Toten“(CRNJANSKI 1993:33) bevölkert wird. Die Begriffe ,Horden“ und ,Scharen' suggerieren, dass es sich hierbei um amorphe Menschenmassen handelt. Mit den unzähligen vereinsamten Frauen sind offenbar Kriegswitwen gemeint, bei den „Horden betrügerischer Händler“ (CRNJANSKI 1993:33) lässt sich an Kriegsprofiteure denken, die Kapital aus dem Elend der breiten Massen ziehen oder bereits gezogen haben.

Die Mobilitätserfahrungen, die der Protagonist während des Ersten Weltkriegs macht, unterscheiden sich in wesentlichen Punkten fundamental von jenen Reiseerfahrungen der Kindheit. Es kann nicht geleugnet werden, dass das östliche Europa bei CRNJANSKI ein höchst mobiler, aber auch multiethnischer und multireligiöser Raum ist. In ihm leben Tschech*innen. Slowak*innen, Pol*innen, Serb*innen, Ungar*innen, Ukrainer*innen, orthodoxe und katholische Christ*innen sowie Juden und Jüdinnen. An dieser Stelle darf jedoch nicht verschwiegen und unterschlagen werden, dass es sich um einen Raum handelt, der u. a. von „schrecklich arme[n]“ und „zerlumpte[n] Juden“ (CRNJANSKI 1993:29) sowie von Mädchen bevölkert wird, die sich offenbar prostituieren müssen. „Mädchen von zwölf Jahren, von zehn Jahren boten sich an“(CRNJANSKI 1993:29).

Nur ganz wenige Textstellen hingegen zeugen von dem kulturellen Reichtum des dargestellten imperialen Raumes. Hier wären exemplarisch die „schönen russischen Kirchen“ (CRNJANSKI 1993:29) zu nennen, aber auch die „Bewunderung für die Melancholie der tschechischen Lieder" (PREVIŠIĆ 2014:32).

Dass wir es deshalb mit einem positiv semantisierten (post-)imperialen Raum zu tun haben lässt sich angesichts der oben angeführten Beispiele schlechterdings nicht behaupten. Das wahrscheinlich eindringlichste Beispiel, mit dem sich dieser Befund untermauern lässt, sind jene ,erhängte[n] Ruthenen“ (CRNJANSKI 1993:25), von denen das Erzähler-Ich berichtet.

Ähnlich wie im Falle der bettelarmen Jüdinnen und Juden sowie der zur Prostitution verdammten Mädchen, sehen sich die Soldaten mit diesem Schreckensszenario während ihrer Märsche durch die galizische Landschaft konfrontiert. 
Im Roman sind keine näheren Informationen darüber zu finden, von wem die Ruthenen gehängt wurden. Es ist dennoch mehr als offenkundig und naheliegend, dass die Verantwortung für diese Gräueltaten beim Habsburger-Imperium liegt. Schließlich ist historisch verbürgt, dass das Habsburger-Reich während des Ersten Weltkriegs besonders brutal gegen Ruthenen vorging (vgl. BORODZIEJ / GÓRNY 2018:255-262).

Nicht das Nationale, sondern das Imperiale entfaltet in CRNJANSKIS Roman sein destruktiv-exkludierendes Potential. So ist zu beobachten, dass nicht die einzelnen Ethnien und Religionen miteinander kollidieren, sondern das Imperium mit seinen ethnisch-religiösen Minderheiten, die ihm allmählich die Loyalität versagen und aufkündigen.

Eine nicht minder befriedigende Interpretation als jene, wonach CRNJANSKI einen imperialen Raum zeichnet, in dem die nationalen Verwerfungen und die Schattenseiten imperialer Herrschaftspraktiken eine eher untergeordnete Rolle spielen, ist indes auch jene, die den Roman als eindeutiges Bekenntnis zum Nationalen, zum Serbischen liest, wie es in Holm Sundhaussens Geschichte Serbiens der Fall ist (vgl. SunDHAUSSEN 2007). CRNJANSKIS Roman Tagebuch über Čarnojević sei integraler Bestandteil des serbischen Nationalmythos geworden, so dass er für die schlechten Beziehungen zwischen Albaner*innen und Serb*innen verantwortlich gemacht werden müsse:

Die ,Erinnerung' an den ,großen Serben-Exodus' von 1690 aus Kosovo nach Südungarn und die nachfolgende Zuwanderung von Albanern nach Kosovo gingen als Bild und Text in den serbischen Nationalmythos ein und vergifteten die serbischalbanischen Beziehungen seit Beginn der Nationsbildung. Erinnert sei stellvertretend an das vielfach reproduzierte Gemälde des Historienmalers Paja Jovanović ,Seobe Srba' (Wanderungen der Serben) von 1896, an den Kurzroman ,Dnevnik o Čarnojeviću“ (Tagebuch über Čarnojević) aus dem Jahr 1921. (SUNDHAUSSEN 2007:51)

Dieser Deutung Sundhaussens liegt insofern ein teleologisches Geschichtsverständnis zugrunde, als eine Kontinuität zwischen dem 19. Jahrhundert und jenen Ereignissen hergestellt wird, die während der neunziger Jahre des 20. Jahrhunderts im ehemaligen Jugoslawien stattfanden. Auf die von Sundhaussen erwähnte Wanderung der Serben wird in CRNJANSKIS Roman zwar verwiesen, doch zeigt sich bei genauerer Betrachtung, dass der Protagonist ein indifferentes Verhältnis zu ihr hat:

Von den Wänden schauten Ikonen auf mich herab, vollbehängt mit Basilikum, da sah man Menschenhaufen, die mit dem Patriarchen an der Spitze auswanderten, und gefesselte Sklaven mit Mädchenaugen. Ich ging zum Spiegel, goß mir lange kaltes Wasser über den Hals und nieste laut. (CRNJANSKI 1993:54) 
Entscheidend ist hier, dass dieses für den serbischen Nationalmythos so bedeutsame Ereignis lediglich mit einem Niesen quittiert wird. Mehrfach werden jedoch auch Schikanen an ethnischen Minderheiten seitens der österreichischungarischen Obrigkeit beschrieben, was den imperialen Raum erneut in einem mehr als fragwürdigen Licht erscheinen lässt. Das gilt für die Zeit vor Ausbruch, aber auch während des Krieges. Doch auch das führt nicht dazu, dass der Protagonist eine eindeutig affirmative Einstellung zum Serbischen entwickelt. Entscheidend ist hier, dass er sowohl zum Nationalen als auch zum Imperialen Distanz wahrt. Seine Reaktion, als er erfährt, dass der österreichisch-ungarische Thronfolger Erzherzog Franz Ferdinand von einem jugoslawischen (jugoslawischer und serbischer Nationalismus waren zu dieser Zeit eins) Nationalisten ermordet wurde, zeugt ebenfalls mehr als deutlich von dessen ambivalenter Haltung dem Nationalen gegenüber:

Im Zug schimpften alle über den Mord. Eine Frau sagte, dieser lächerliche Held vom Sankt Veitstag sei verkommen, wie alle Gymnasiasten und Gymnasiastinnen in Sarajevo verkommen seien. Meine Augen waren voll Tränen. ${ }^{1}$ (CRNJANSKI 1993:10)

Die Tränen könnten hier als Bekenntnis zum Nationalen, in diesem Falle zum Serbischen, und als Abgrenzung vom Imperialen gedeutet werden. Der Protagonist weint schließlich nicht um irgendjemanden, sondern um Gavrilo Princip und damit um einen serbischen Nationalisten. Bei genauerer Lektüre wird jedoch einsichtig, wie gebrochen und komplex die Einstellung zum vermeintlich Eigenen, zum Nationalen tatsächlich ist. Nicht von ungefähr heißt es sogleich: „Ach, ich war damals jung, so jung" (CRNJANSKI 1993:11). Das zweimal wiederholte Adjektiv jung, das noch durch das Partikel „so“ und die Interjektion „Ach“ verstärkt wird, erhellt, dass es sich hier um die desillusionierte, ja defätistische Perspektive eines aus dem Krieg zurückgekehrten handelt, der resigniert und mit Distanz auf seine Jugend, aber auch auf seine Nation blickt. Doch auch dem Habsburger-Reich steht der Protagonist mehr als kritisch gegenüber. Nachdem

Mit dem als lächerlich bezeichneten Helden ist der serbisch-jugoslawische Nationalist Gavrilo Princip gemeint, der am 28. Juni 1918 - am St. Veitstag - den österreichischen Thronfolger Franz Ferdinand und dessen Gemahlin in Sarajevo erschoss. Der St. Veitstag hat insofern eine besondere historische Bedeutung, als die „Serben“ im Jahre 1389 eine vernichtende Niederlage gegen das Osmanische Reich erlitten. Auch wurde im Jahre 1921 die Vidovdan-Verfassung für den ersten jugoslawischen Staat (das Königreich der Serben, Kroaten und Slowenen) am St. Veitstag verabschiedet. 
der österreichisch-ungarische Thronfolger von einem serbischen Nationalisten ermordet wurde, wird die Reaktion des Imperiums wie folgt geschildert:

Später sah ich den Hof voll von Popen. Männern und Frauen. Oh, wie war das lächerlich. Wir mußten alle die Nase an die Wand drücken und stillschweigen. So stand ich bis zum Abend. Dann fiel ich in Ohnmacht. Ich war ein sehr zarter junger Herr (CRNJANSKI 1993:11).

Hier wird die serbische Minderheit dafür verantwortlich gemacht und zur Rechenschaft gezogen, dass der Thronfolger von einem Serben ermordet wurde. Die Schikanen gehen soweit, dass potentiell jeder der Spionage bezichtigt wird:

Ein Pope lag neben mir; sein Mund blutete, und die Zähne waren ihm eingeschlagen. Dann führten sie eine junge Frau mit zwei Kindern herein, und es war schamlos, wie sie mit ihr umgingen. Auch sie schrien sie an: ,Spion, Spion!' Sie, ganz bleich, liebkoste die kleinen, braunen Köpfe ihrer Kinder. (CRNJANSKI 1993:11f.)

Diese Ungleichbehandlung von Mitgliedern kleinerer Nationen setzt sich im Militär, d. h. während des Ersten Weltkrieges fort. Auch hier wird von Schlägen und Beschimpfungen berichtet. Dass die Schmähungen und Anpöbelungen dabei von deutsch und ungarisch sprechenden Vorgesetzten artikuliert werden, ist alles andere als Zufall:

Aus einem Loch ruft mir ironisch einer auf ungarisch zu und flucht abscheulich. Rechts von uns kommen wie Ameisen die Schwärme des dreiunddreißigsten Regiments aus den Gräben und stürmen mit schrecklichem Gebrüll, Rufen und Wehklagen. Wir winden uns durch den Stacheldraht. Einer lästert auf deutsch die Mutter und nennt uns Hunde. (CRNJANSKI 1993:22)

Das Regiment, in dem der Protagonist dient, wird höchst abfällig als „Hundsregiment" (CRNJANSKI 1993:22) bezeichnet. Weitaus weniger blutige und martialische Züge trägt das südöstliche Europa, das JOSEPH ROTH in seinen Reportagen Reise auf dem westlichen Balkan zeichnet. Dies ist vor allem der Tatsache geschuldet, dass RоTH die Zeit nach dem Ersten Weltkrieg darstellt.

ROTH verfasste diese Texte 1927 für die FZ (vgl. LUNZER:156-198). ${ }^{2}$ In dieser Sammlung von Reportagen schreibt er über Albanien, Jugoslawien, über Städte wie Sarajevo, Belgrad und Tirana. Ich greife hier seine Reportage Blick nach Südslawien (ROTH 2013a:262-265)und Wo der Weltkrieg begann (ROTH 2013b:259-261) heraus. In diesem Text beobachtet RоTH - genauer das berichtende Ich - auf scharfsinnige Weise, in welcher Form sich die diversen kulturellen und historischen Traditionen in diesem Teil Europas sedimentiert

2 Über die Entstehungsgeschichte von RотнS Reportagen sowie die Gründe für RотнS Reisen auf den Westbalkan informiert ausführlich der oben angeführte Aufsatz von Heinz Lunzer. 
haben. Dabei wird die nationale Ebene immer wieder in eine (post-)imperiale Perspektive gerückt. Über Belgrad heißt es:

Man nennt Belgrad das Paris des Balkans. Lippenstifte, Wachsbüsten, Haarschnitte und Damenmoden, die Einrichtung der Kaffeehäuser und der Restaurants, der Geschmack der Hors d'œuvres und der Aperitifs, eine ganz bestimmte Sorglosigkeit in der Atmosphäre des abendlichen Korsos, ein ganz bestimmter Glanz über jenem heiter bewegten Straßenbild, das man ,Leben und Treiben nennt", eine gewisse phosphoreszierende Oberfläche: all das ist Pariser Konvenienz. Es gibt auch eine französische Buchhandlung - allerdings. Aber führte sie nicht den ,Sourire‘ auf Lager, man könnte von einem geistigen französischen Einfluss überhaupt nicht sprechen. Dekobra, Claude Anet und andere von der französischen Schriftstellersorte, die den literarischen Qualitätsexport Frankreichs bildet und den geistigen Charakter ihres Landes fälscht, während sie es in fernen Gegenden repräsentiert, verleihen ihren jugendlichen Lesern die bekannte pseudofranzösische Haltung, deren Kennzeichen Unbeständigkeit, Nachtleben-Erotik und Kinogalanterie sind. (RоTн 2013a:262)

Für das Verständnis der Semantisierung, d. h. Bewertung des (süd-)östlichen Europas in RоTHS Reportagen über den Westbalkan ist zentral, dass die französischen und damit nationalen Einflüsse westeuropäischer Provenienz auf ehemals imperialen Raum immer stärker sichtbar werden. Daran ändert auch die Tatsache nichts, dass es sich bei all jenem, was als spezifisch französisch wahrgenommen wird, letztlich um literarisch vermittelte Klischees handelt, die aus der Perspektive des Erzähler-Ichs nichts mit Frankreich zu tun haben.

In diesem Kontext ist bezeichnend, dass der Schriftsteller Claude Anet (bürgerlich Jean Schopfer) genannt wird; ein französischer Autor Schweizer Herkunft, der u.a. Romane über russische Frauencharaktere (Ariane, jeune fille russe) schrieb.

Im gleichen Atemzug wird aber das Bild eines Polizeistaates, einer Diktatur gezeichnet, die keinerlei Vertrauen zu der eigenen Bevölkerung aufzubringen imstande ist. Den Bewohner*innen selbst werden hingegen keine negativen, sondern, so erscheint es zumindest auf den ersten Blick, positive Eigenschaften zugeschrieben

Indessen ist gerade das südslawische Volk intelligent, aufgeweckt, diszipliniert, politisch selbstständig, kritisch, mit einem hellen, gesunden, ländlichen Verstand begabt, human, heiter, kultiviert, von einer guten, südlichen Sonne gesegnet, ohne nationalistische Vorurteile, ohne jeden religiösen Fanatismus, loyal gegen andere Nationen, Stämme und Rassen. (RоTн 2013a:264)

Zieht man jedoch in Betracht, dass die Südslawen, genauer Jugoslawen, mit „einem hellen, gesunden, ländlichen Verstand“ ausgestattet werden, so zeigt sich, dass das berichtende Ich auf diese Weise neue Stereotype produziert. Mit 
der Formulierung ,ländliche[r] Verstand“ wird sowohl auf die mangelnde Urbanisierung als auch auf eine gewisse Einfältigkeit der Menschen im Vergleich zu Westeuropa angespielt.

In RoTHS Reportage Wo der Weltkrieg begann wird Sarajevo ebenfalls vor der Folie nationaler und imperialer Spuren in den Blick genommen. Sarajevo wird vor diesem Hintergrund, und hier zeigt sich ebenfalls, wie sehr ROTH die Gefühle seiner Leser*innen zu aktivieren sucht, als ,[u]nschuldige, aber fluchbeladene Stadt" und als ,[t]raurige Hülle der schauderhaftesten Katastrophe“ (ROTH 2013b:259) dargestellt. Schließlich wird durch das Attentat auf den österreichischungarischen Thronfolger Erzherzog Franz Ferdinand das Ende des Habsburger Reiches, des Osmanischen Reiches und des Russischen Zarenreiches im Zuge des Ersten Weltkriegs endgültig besiegelt. Sarajevo kann für den Erzähler, der nostalgisch auf das untergegangene Habsburgerreich zurückblickt, daher nur auf den ersten Blick eine gewöhnliche Stadt sein:

Es gibt ein Theater, man spielt eine Oper, es gibt ein Museum, es gibt Spitäler, einen Magistrat, Polizisten, alles, was eine Stadt brauchen kann. Eine Stadt! Als wäre Sarajevo eine Stadt wie jede andere! Als hätte in Sarajevo nicht der größte aller Kriege angefangen. (ROTH 2013b:261)

Eine ,normale' Stadt kann Sarajevo für den Erzähler aber vor allem deshalb nicht sein, weil ,alle Heldengräber, alle Massengräber, alle Schlachtfelder, alle Giftgase, alle Krüppel, alle Kriegswitwen, alle unbekannten Soldaten“ (ROTH 2013b:261) in dieser Stadt ihren Ursprung hätten. Sarajevo steht damit metonymisch für die Schrecken und Gräuel des Ersten Weltkriegs. Die einzige Konsequenz, die der Erzähler aus diesem Befund zu ziehen vermag, ist die, dass Sarajevo zwar nicht untergehen, aber ,allen zum schrecklichen Gedächtnis“ (ROTH 2013b:261) dienen müsse. Die Stadt dürfe nicht zerstört werden, weil sie „gute, liebe, schöne Frauen“ und „wunderbar unschuldige Kinder“ (ROTH 2013b:261) habe. Wie in Belgrad dominieren nicht mehr die imperialen, sondern die französisch-westeuropäischen Einflüsse das Stadtbild. Und ähnlich wie in Belgrad sind sie in Form literarischer Erzeugnisse und Exporte greifbar:

Ein Buchhändler verkauft Papier und Bücher und literarische Zeitschriften - aber mehr zu repräsentativen Zwecken. Ich erstehe bei ihm einen Maupassant (obwohl er schon Dekobra auf Lager hat) für eine Nacht im Zug ohne Schlafwagen. (Rотн 2010b:260)

Der Reisende entscheidet sich hier offenbar bewusst gegen Maurice Dekobra, der eigentlich Maurice Tessier hieß, und kauft ein Buch von Maupassant. Dekobra ist genau wie Anet, auf den in der Reportage Blick nach Südslawien verwiesen wurde, ein Repräsentant französischer Unterhaltungsliteratur. Das berichtende 
Ich protestiert mit der Ablehnung Dekobras gegen Verbreitung derartiger Literatur. Neben diesem Einfluss französischer Literatur spielen, wie sollte es für Bosnien auch anders sein, osmanische und habsburgische Hinterlassenschaften eine prägende Rolle. Sie haben sowohl in der Sprache, dem Kleidungsstil der Bewohner, aber auch in der Architektur ihren Niederschlag gefunden. Die osmanischen Erbschaften werden jedoch lediglich durch „einen kleinen türkischen Friedhof" (ROTH 2013b:260) und den ,orientalische[n] Bazar“ (ROTH 2013b:260) repräsentiert.

Das Erbe Österreich-Ungarns hingegen hat seine Spuren in Gestalt eines Dienstmanns, der einen „Backenbart“ trägt, sowie „,[g]anz alte Männer, wahrscheinlich Notare“ (ROTH 2013b:260) hinterlassen, die ,außer Dienst“" (ROTH 2013b:260) sind. Wie sehr die durch sie repräsentierte imperiale Tradition ein Relikt der Vergangenheit ist, wird an der Bemerkung, dass sie „das ärarische Deutsch aus österreichischen Zeiten“ (ROTH 2013b:260) sprechen, sehr deutlich. Und auch die Tatsache, dass der Backenbart des Dienstmanns als Gespenst aus der Doppelmonarchie“" (ROTH 2013b:260) bezeichnet wird, zeigt überdeutlich, wie sehr das Imperiale von nationalen Entwicklungen und Einflüssen überlagert wurde.

Die Komplexität der Raumdarstellungen und -semantisierungen in CRNJANSKIS Roman und ROTHS Reportagen entsteht erst dadurch, dass Nationales und Imperiales auf eine höchst spannungsgeladene Weise zueinander in Beziehung gesetzt werden. Darin liegt letztlich auch das palimpsestartige des südöstlichen und des östlichen Europas in CRNJANSKIS Roman und ROTHS Reportagen über den Westbalkan begründet. (Post-)imperiale Räume, wie sie bei ROTH und CRNJANSKI dargestellt werden, sind weder Orte des friedlichen Miteinanders noch können sie einseitig als Völkerkerker betrachtet und verdammt werden. CRNJANSKIS Tagebuch und ROTHS Reportagen entziehen sich derartig eindeutigen Zuschreibungen. Dies stellt die eingangs vorgestellten kulturwissenschaftlichen Thesen in Frage, wonach (post-)imperiale Räume als Modell für ein übernationales Europa - jenseits nationalstaatlicher Egoismen - dienen können. In einem nicht unwesentlichen Punkt ist der kulturwissenschaftlichen Imperien-Forschung indes zuzustimmen.

Weder bei CRNJANSKI noch bei ROTH wird die ethnisch-religiöse Heterogenität imperialer Räume dafür verantwortlich gemacht, dass Imperien zerfallen. Eine Beschreibung (post-)imperialer Räume in der Literatur kommt daher nicht umhin, die vielfältigen Konstellationen zu untersuchen, die sich in der Gleichzeitigkeit der Ungleichzeitigkeit historischer Prozesse, auch und besonders in imperial und postimperial geprägten Räumen, niedergeschlagen haben. Nationales und (Post-)Imperiales müssen in diesem Kontext noch viel stärker als aufeinander 
bezogene und sich nicht per se ausschließende Phänomene gedeutet werden. Und was immer unter ,national' im Einzelnen auch zu verstehen ist, lässt sich die Semantisierung imperialer und postimperialer Räume, jedenfalls mit Blick auf das 19. und 20. Jahrhundert, ohne dessen Berücksichtigung schlechterdings nicht denken und beschreiben.

\section{Literatur}

Bobinac, Marijan / Chovanec, Johanna (eds.) (2018): Postimperiale Narrative im zentraleuropäischen Raum. Tübingen.

Borodziej, WŁodzimierz / Gorny, MACIEJ (2018): Der vergessene Weltkrieg. Europas Osten 1912-1923. Bd 1: Nationen. Stuttgart.

Burbank, JAne / CoOPER, Frederick (2010): Empires in World History. Princeton.

CRnjanski, MiLoš (1993): Dnevnik o Čarnojeviću / Tagebuch über Čarnojević [1921]. Aus dem Serbischen von Hans Volk. Berlin.

CSÁKY, MORITZ (2009): Zentraleuropa im Spannungsfeld kultureller Kommunikationsräume, In: Ulbrecht, Siegfried / UlbréChtova, Helena (eds.): Die Ost-West-Problematik in den europäischen Kulturen und Literaturen. Dresden, 53-75.

CÁKY, MORITZ (2010): Das Gedächtnis der Städte. Kulturelle Verflechtungen - Wien und die urbanen Milieus in Zentraleuropa. Wien, 36-78.

Grob, Thomas / Pevišić, Boris (eds.) (2014): Erzählte Mobilität im östlichen Europa. (Post-)Imperiale Räume zwischen Erfahrung und Imagination. Tübingen.

HiRSCHHAUSEN, ULRIKE VON / LEONHARD, JÖRN (2011): Empires und Nationalstaaten. Freiburg.

HiRSCHHAUSEN, UlRIKE VON / LEONHARD, JÖRN (2012): Comparing Empires: Encounters and Transfers in the Long Nineteenth Century. Freiburg.

Koselleck, ReInHART (2015): Zeitschichten. Studien zur Historik. Frankfurt a. M.

KÜPPER, ACHIM (2010): Berichte aus der Fremde. Unbehaustheit als Grundmotiv von Joseph Roths Reportagen und Reiseschilderungen. In: EICHER, THOMAS (ed.): Joseph Roth und die Reportage. Heidelberg, 99-125.

LunZER, HeINZ (2016): Joseph Roths Reise nach ,, Südslawien “ und Albanien im Jahr 1927. In: PESnel, StÉPHANe / TunNer, ERIKA (eds.): Joseph Roth - Städtebilder. Zur Poetik, Philologie und Interpretation von Stadtdarstellungen aus den 1920er und 1930er Jahren. Berlin, 156-198.

Müller-FunK, WolfGang (2012): Joseph Roth. Wien.

MÜNKLER, HERFRIED (2005): Imperien. Die Logik der Weltherrschaft-vom Alten Rom bis zu den Vereinigten Staaten. Berlin. 
Orosz, Magdolna (2018): ,,Raum “ und Raumdarstellung als Kategorien literarischer (narrativer) Textanalyse. Annäherungen und Fallbeispiele. In: DÁCZ, ENIKÖ (ed.): Räumliche Semantisierungen. Raumkonstruktionen in den deutschsprachigen Literaturen aus Zentral- und Südosteuropa im 20. und 21. Jahrhundert. Regensburg, 7-35.

OSTERHAMMEL, JÜRGEN (2006): Imperien im 20. Jahrhundert. Eine Einführung. In: Zeithistorische Forschungen / Studies in Contemporary History. Online-Ausgabe, 3 (2006), H. https://zeithistorische-forschungen.de/1-2006/4627 (5.11.2019). Druckausgabe:4-13.

Osterhammel, JÜrgen (2010²): Imperien. In: Budde, GunNila / CONRAD, SEBASTIAN (eds.): Transnationale Geschichte. Themen, Tendenzen und Theorien. Göttingen, 56-67.

OSTHUES, JULIAN (2017): Interkulturelle Metaphern. Überlegungen zu ihrer Theoretisierung am Beispiel des Palimpsests. In: Zeitschrift für interkulturelle Germanistik 8, H. 2:37-52.

PATEL, KLAUS KIRAN (2008): Überlegungen zu einer transnationalen Geschichte [2004]. In: Osterhammel, JÜRGEN (ed.): Weltgeschichte. Basistexte. Stuttgart, 67-89.

PREVIŠIĆ, BORIS (20014): Literarische Erinnerungen an das Imperium als Utopie. Die historische Zäsur des Ersten Weltkriegs. In: GROB, THOMAS / PREVIŠIĆ, BORIS (eds.): Erzählte Mobilität im östlichen Europa. (Post-)Imperiale Räume zwischen Erfahrung und Imagination. Tübingen, 25-42.

Roth, JosePH (2013a): Blick nach Südslawien [1927]. In: NüRnBERGER, HeLmuTH (ed.): Joseph Roth. Ich zeichne das Gesicht der Zeit. Essays, Reportagen, Feuilletons. Zürich, 262-265.

ROTH, JosEPH (2013b): Wo der Weltkrieg begann [1927]. In: NÜRNBERGER, HELMUTH (ed.): Joseph Roth. Ich zeichne das Gesicht der Zeit. Essays, Reportagen, Feuilletons. Zürich, 259-261.

SCHLÖGEL, KARL (2003): Im Raum lesen wir die Zeit. Über Zivilisationsgeschichte und Geopolitik. München.

Sundhaussen, Holm (1999): Europa balcanica. Der Balkan als historischer Raum Europas. In: Geschichte und Gesellschaft 25, H. 4:638-651.

SundHaussen, Holm (2007): Geschichte Serbiens: 19.-21. Jahrhundert. Wien.

SÜltemeyer-Von LiPS, IngEBorg (2016): ,Städte haben viele Gesichter“. Wieviel Fiktion vertragen Reportage und Feuilleton? In: PESNEL, STÉPHANE / TUNNER, ERIKA (eds.): Joseph Roth - Städtebilder. Zur Poetik, Philologie und Interpretation von Stadtdarstellungen aus den 1920er und 1930er Jahren. Berlin, 31-59

THER, PHILIPP: Von Ostmitteleuropa nach Zentraleuropa - Kulturgeschichte als Area Studies, In: H-Soz-Kult, 02.06.2006 www.hsozkult.de/article/id/artikel-739> (5.11.2019),

TODOROVA, MARIA (2002): Der Balkan als Analysekategorie: Grenzen, Raum, Zeit. In: Geschichte und Gesellschaft 28, H. 3:470-492.

TODOROVA, MARIA (2003): Historische Vermächtnisse als Analysekategorie. Der Fall Südosteuropa. In: KASER, KARL (ed.): Europa und die Grenzen im Kopf. Klagenfurt, 89-111. 
Jenseits von Völkerkerker und friedlichem Miteinander: Semantisierungen...

TODOROVa, Maria (2007): Die Kategorie der Zeit in der Geschichtsschreibung über das östliche Europa. Oscar-Halecki Vorlesung 2003. Leipzig.

Waltl, LuKas (2016): Die „große Vermessenheit, Städte beschreiben zu wollen “. In: Pesnel, StéPhane / Tunner, ERIKA (eds.): Joseph Roth - Städtebilder. Zur Poetik, Philologie und Interpretation von Stadtdarstellungen aus den 1920er und 1930er Jahren. Berlin, 1-30. 